\title{
Point Defects in Magnesium Aluminates Spinel Ceramics Doped with Lithium Fluoride
}

\author{
V.T. Gritsyna ${ }^{a, *}$, Yu.G. Kazarinov ${ }^{a}$, A.O. Moskvitin ${ }^{a}$ And I.E. Reimanis ${ }^{b}$ \\ ${ }^{a}$ Department of Physics and Technology, V.N. Karazin Kharkiv National University \\ Svoboda Sq. 4, 61077 Kharkiv, Ukraine \\ ${ }^{b}$ Colorado Center for Advanced Ceramics, Metallurgical and Materials Engineering Department \\ Colorado School of Mines, Golden, CO 80401, USA
}

\begin{abstract}
The nature and topological distribution of optical centers within various regions of hot pressed disks of transparent magnesium aluminates spinel ceramics doped with LiF were studied. In the optical absorption spectra of this type of ceramic, bands were revealed at $4.75 \mathrm{eV}$ and $5.3 \mathrm{eV}$, which were identified with $\mathrm{F}^{+}-$and F-centers, respectively. Because both bands are formed by anionic vacancies which captured one or two electrons, the topological distribution of anionic vacancies was determined. The band at $5.65 \mathrm{eV}$ was also found which is tentatively identified with complex centers of anionic vacancies that capture fluorine ions and electrons. Using X-ray irradiation the variety of absorption bands of hole centers related to cationic vacancies was established. The spatial distribution of cationic vacancies within the ceramic disk was also determined.
\end{abstract}

PACS numbers: 81.05.Je, 61.72.J-

\section{Introduction}

Magnesium aluminates spinel, $\mathrm{MgAl}_{2} \mathrm{O}_{4}$ (hereafter termed "spinel"), shows excellent optical transmittance at the wavelength of $3-5 \mu \mathrm{m}$ and currently is a leading candidate for infrared missile domes and windows [1]. Also it has high hardness, strength, and chemical resistance, and therefore it has potential applications as armour [2]. Because single crystals are restricted in size and their production is very expensive, the development of ceramic fabrication technology of this kind of material is a critical need. There have been many attempts to produce transparent spinel ceramics of pure spinel, but only some of them have been successful. Conditions to achieve parts with no light scattering pores, second phases or defects are difficult to achieve - this may be due to a combination of reasons, including the alteration of the spinel powder starting composition and the formation of defects and their clusters $[3,4]$.

One of the most successful processes for producing high optical quality spinel ceramics is hot-pressing of spinel powder doped with $\operatorname{LiF}[5,6]$. Variations in the processing conditions have been correlated to changes of microstructure, porosity, optical and mechanical properties of spinel ceramics. Backscattered electron (BSE) images of the fractured surfaces of these ceramics obtained by

\footnotetext{
* corresponding author; e-mail:

gritsyna@pht.univer.kharkov.ua
}

the present authors indicate that there exists a variation of the microstructure through the body of monolith. Based on earlier electron microscopy studies, these microstructure variations correlate with variation of point defects and impurities in the lattice [7]. Investigations of sintering kinetics of spinel doped with LiF show that the presence of LiF lowers the effective activation energy for the densification of spinel [8]. Based on TEM observations it was proposed that oxygen vacancies are generated by a mechanism that originates via the presence of LiF.

In this paper, using optical spectroscopy, we investigate the nature of lattice point defects and their topological distribution throughout disks of transparent spinel ceramics doped with $\mathrm{LiF}$ up to 1.0 wt.\%.

\section{Experimental details}

Spinel ceramics were prepared by a hot-pressing technique in the form of disks $50 \mathrm{~mm}$ in radius and $7 \mathrm{~mm}$ in thickness. The ceramic was produced by hot-pressing of spinel powder $\mathrm{MgAl}_{2} \mathrm{O}_{4}: \mathrm{LiF}$ (1 wt.\%) at $1550^{\circ} \mathrm{C}$, at $35 \mathrm{MPa}$ in vacuum environment. From the disk a segment of circular arc of $35 \mathrm{~mm}$ height was cut and cracked into two pieces, so that BSE scanning electron microscopy could be performed on the fracture surfaces, as shown in Fig. 1. The samples for optical investigations were cut from this segment, such that we could search for differences in optical properties along the radius from center to periphery. Slices $0.7 \mathrm{~mm}$ in thickness were polished to optical finish. It was revealed that the slices were optically inhomogeneous in a radial direction 
(Fig. 2). Near the arc rim there is a transparent area (a); further, towards the center of the slice exists a smoke-colored strip (b) which transforms in a diffusive $U$-type shape with a transparent area (c) inside the U shape.

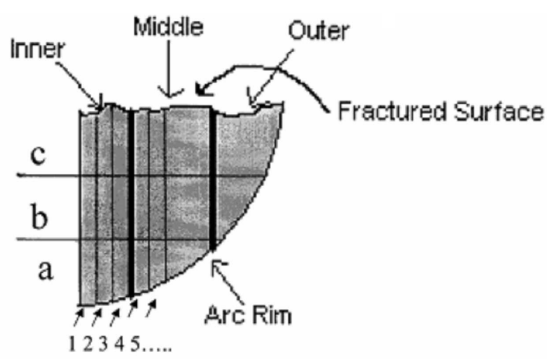

Fig. 1. The part of ceramic disk from which the specimens for investigations were cut.

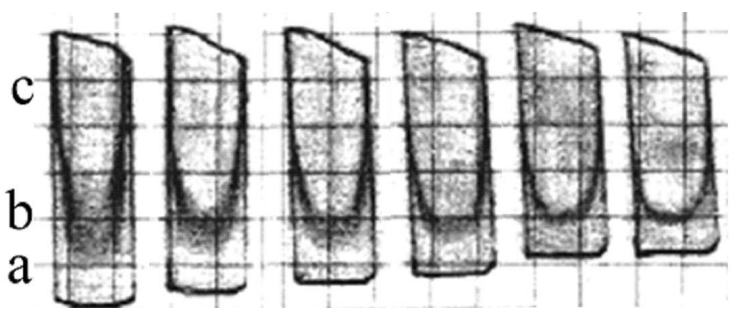

Fig. 2. The cut portion of transparent ceramic samples.

Measurements of the optical absorption spectra were provided with a single beam SF-46 spectrophotometer in the wavelength range of $186-960 \mathrm{~nm}(1.3-6.6 \mathrm{eV})$. We measured the optical absorption over the length of strips (along the radius) in spots (a), (b), and (c) to obtain information on the topological distribution, the nature and concentration of point defects in the ceramic disc. Also, we provided measurements of optical absorption in spinel ceramics irradiated with X-rays to activate latent defects in pristine samples into optical centers.

\section{Results and discussion}

\subsection{Optical absorption centers and defects in pristine samples of spinel ceramics}

The typical optical absorption spectra for some samples measured in spots (a), (b), and (c) (along radius) are presented in Fig. 3. Because the thickness of all samples was the same, the data on absorption is presented in relative units. Absorption spectra of the most transparent area near the arc rim, region (a), have one band in the vicinity of photon energy of $5.5 \mathrm{eV}$. Absorption in UV-region $(>6 \mathrm{eV})$ is mainly associated with uncontrolled impurity ions or defined by the quality of polishing. For this region there is no significant difference in absorption spectra for different samples.

Optical absorption spectra of the cloudy (smoke-colored) region (b), are very different from sample to

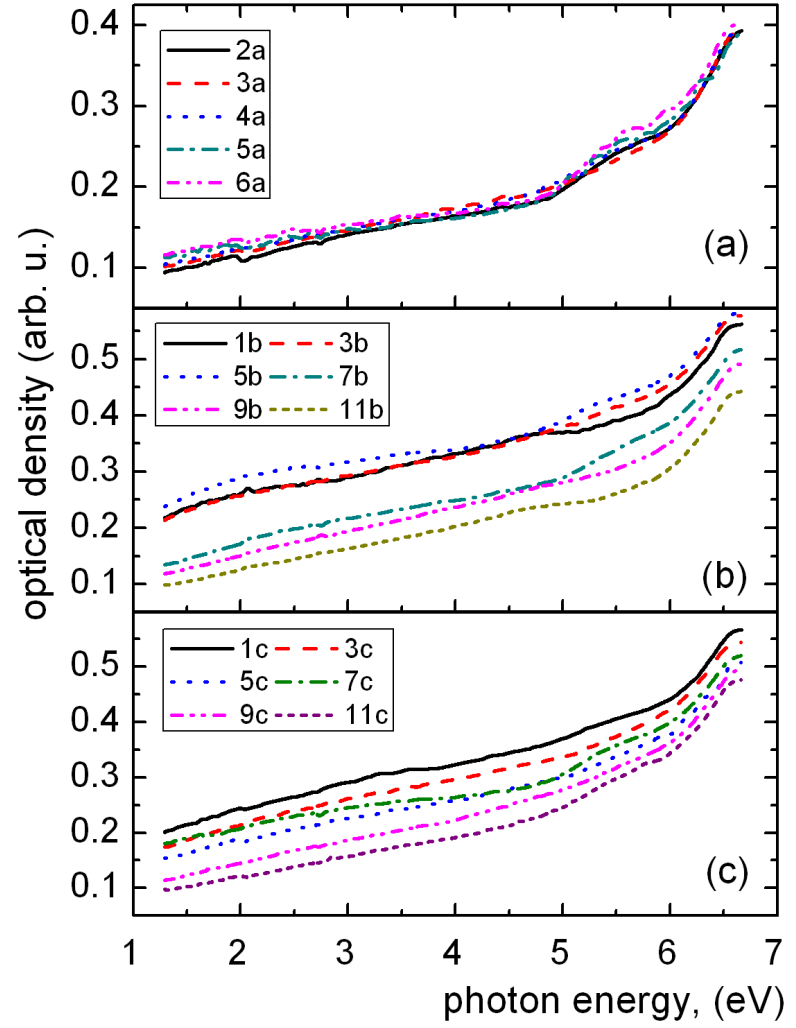

Fig. 3. Typical absorption spectra of some ceramic samples in spots (a), (b), and (c) which were cut from the sector of ceramic disk (Fig. 1).

sample (Fig. 3b). From visual pictures of the samples one can see that shape of cloudiness spot varies from sample to sample, and therefore a diaphragm of spectrophotometer, which covers spot of $4 \times 6 \mathrm{~mm}^{2}$, should include regions of different cloudiness. In these spectra one can distinguish weak bands at $4.75 \mathrm{eV}$ and about $5.3 \mathrm{eV}$.

Finally, for the central transparent part of the samples, region (c), absorption spectra do not show any prominent bands. Furthermore, optical density decreases toward the periphery. To deconvolute specific absorption bands on the strong background (which we propose is due to scattering at grain boundaries), we subtract the optical absorption spectra obtained for different samples in the same spot (a, b, or c). As an example, the difference spectra are shown in Fig. 4. In the area (a) the difference spectra show the presence of a band at $5.65 \mathrm{eV}$, the intensity of which varies slightly from sample to sample. Also, the band at $4.75 \mathrm{eV}$ exhibits intensity which varies in an opposite manner to that of the band at $5.3 \mathrm{eV}$ (i.e., in the spectrum we see not a peak but a hole). In the absorption spectra of the area (b) the main absorption band has the maximum at photon energy of $4.75 \mathrm{eV}$. Finally, absorption spectra in area (c) reveal the main band at $5.3 \mathrm{eV}$, the intensity of which decreases towards periphery. 


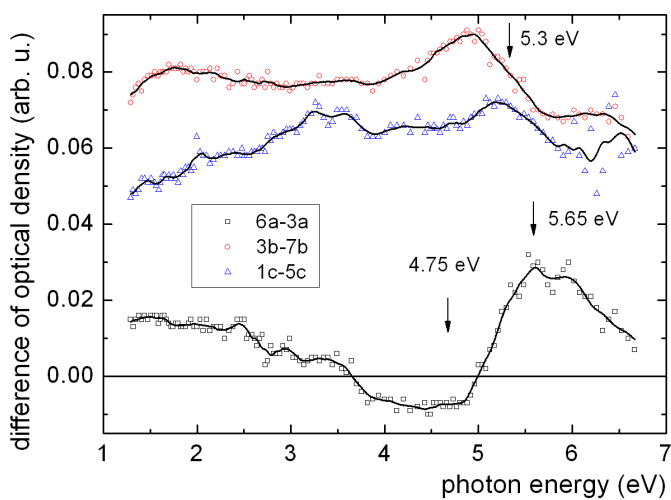

Fig. 4. Typical difference absorption spectra in spots (a), (b), and (c) for some ceramic samples cut from the sector of ceramic disk.

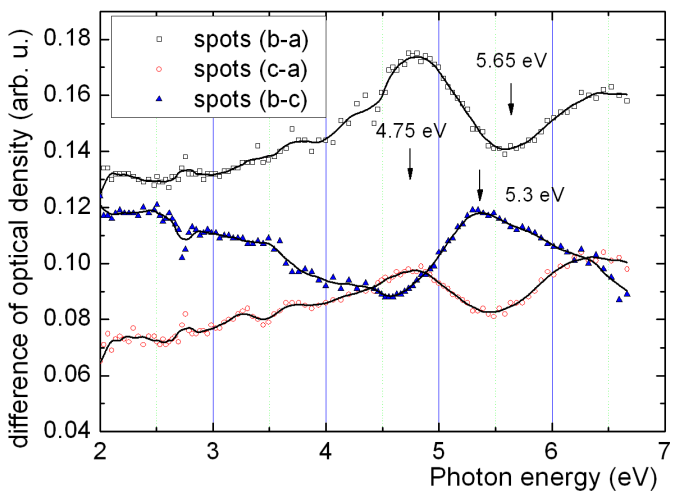

Fig. 5. Typical difference absorption spectra of spots (b) and (a), (c) and (a), (b) and (c) for transparent ceramics $\mathrm{MgAl}_{2} \mathrm{O}_{4}: \mathrm{LiF}$.

The comparison of absorption spectra in one specimen but in different spots also shows the existence of indicated three bands. As an example, in Fig. 5 we present the difference spectra between (b) and (a), (c) and (a), (b) and (c) for one of the samples. This difference of optical density includes also the light scattering, which is different for (a), (b), and (c) spots. Therefore, the positive difference of optical density is due to larger contribution of light scattering. Also, as mentioned above, in the difference spectra the existence of selective absorption bands can be manifested as a peak and as a hole. From Fig. 5 we can conclude that the intensity of the band at $4.75 \mathrm{eV}$ in spot (b) compared with spot (a) increases, but that of the band at $5.65 \mathrm{eV}$ decreases. A similar difference in spectra is observed for spots (c) and (a). Additionally, the absolute values of this latter difference is smaller compared with that of (b-a) spots. The difference spectra of spots (b-c) demonstrate a decrease of the $4.75 \mathrm{eV}$ band but an increase of the band at $5.3 \mathrm{eV}$, together with some contribution of the band at $5.65 \mathrm{eV}$.

The previous detailed investigations of the origin of defects and optical centers in spinel single crystals al- low us to correlate the observed absorption bands with appropriate defects [9]. The band at $4.75 \mathrm{eV}$ was identified with $\mathrm{F}^{+}$-centers which represent anion vacancies that have captured one electron. A free anion vacancy has no specific absorption band in the visible region of the spectra. Therefore, during the densification of spinel, the creation of anion vacancies takes place. Also, at high temperature a free electron can be generated and captured by an anion vacancy, thereby forming the $\mathrm{F}^{+}$center. Another band at $5.3 \mathrm{eV}$ has been well-studied and identified with the F-center, comprising an anion vacancy that has captured two electrons. The mechanism of formation of these two types of centers is similar. The last band at $5.65 \mathrm{eV}$ was not observed in absorption spectra of spinel single crystals and spinel ceramics prepared without doping with LiF. Therefore, the mechanism of formation of this band could be related to solid chemical reaction between spinel $\mathrm{MgAl}_{2} \mathrm{O}_{4}$, and LiF. Analysis of reaction between $\mathrm{MgAl}_{2} \mathrm{O}_{4}$ and $\mathrm{LiF}$ leads to the conclusion that oxygen vacancies are formed due to the incorporation of both $\mathrm{Li}$ and $\mathrm{F}$ ions into crystal lattice [8]:

$$
3 \mathrm{LiF} \stackrel{\mathrm{MgAl}_{2} \mathrm{O}_{4}}{\longrightarrow}\left(\mathrm{Li}_{\mathrm{Mg}}\right)^{-}+2\left(\mathrm{Li}_{\mathrm{Al}}\right)^{2-}+3\left(\mathrm{~F}_{\mathrm{O}}\right)^{+}+\mathrm{V}_{\mathrm{O}}^{2+}
$$

The residual oxygen vacancies $\left(\mathrm{V}_{\mathrm{O}}^{2+}\right)$ can capture one or two electrons forming $\mathrm{F}^{+}$- or F-centers, consistent with the observation of absorptions bands at $4.75 \mathrm{eV}$ and $5.65 \mathrm{eV}$. Other products of this reaction, such as fluorine ions in oxygen sites $\left(\mathrm{F}_{\mathrm{O}}\right)^{+}$, may serve as electron traps forming electron centers, which could be responsible for the absorption band at $5.65 \mathrm{eV}$.

\subsection{Optical absorption centers in irradiated samples of spinel ceramics}

Irradiation with X-rays was provided using X-ray tube of $\mathrm{Cu}$ anode operated at $40 \mathrm{kV}$ and current up to $0.4 \mathrm{~mA}$ during $1.5 \mathrm{~h}$. This time was sufficient to reach saturation in radiation-induced absorption. As an example, the initial absorption spectra and spectra of the X-ray irradiated sample for different spots are presented in Fig. 6. One can notice the increase of absorption in irradiated material for all spots in the spectral range $2-6 \mathrm{eV}$. For detailed analysis of radiation induced absorption we subtract spectra of the initial sample from the spectra of the irradiated one (Fig. 7). Evidently, for all spots the radiation-induced absorption reveals several highly overlapped bands at photon energies of $2.8 \mathrm{eV}, 3.3 \mathrm{eV}$, and $3.8 \mathrm{eV}$.

It was revealed that in many oxide crystals, including constituent $\mathrm{MgO}$ and $\mathrm{Al}_{2} \mathrm{O}_{3}$ oxides of studied crystals, in the spectral range of $2-4 \mathrm{eV}$ under irradiation there appeared wide absorption bands which are related to hole centers. Hole centers represent cation vacancies in octahedral $\left(\nu_{c}^{3-}\right)$ or in tetrahedral $\left(\nu_{c}^{2-}\right)$ positions of effective charges three or two mines, respectively, which have captured one or two holes forming $\mathrm{V}^{=}$- or $\mathrm{V}^{-}$-centers depending on the net negative charge of cation vacancies. Capture is related to nearby oxygen ions. In irradiated spinel single crystals in this spectral range we 


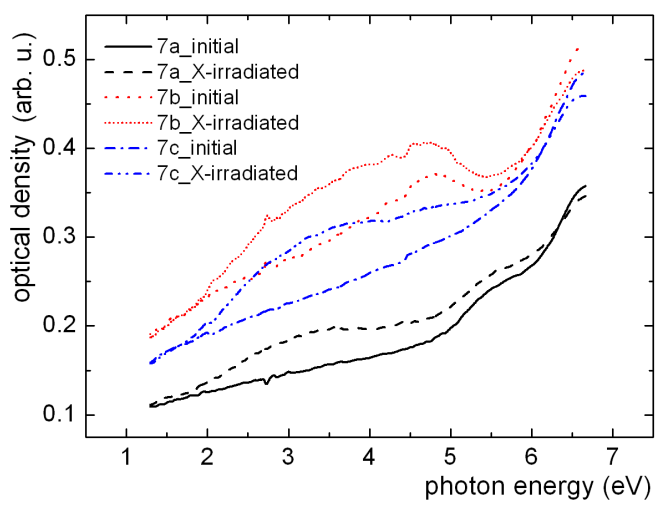

Fig. 6. Typical absorption spectra of initial and $\mathrm{X}$-irradiated samples in different spots (a), (b) and (c) for transparent ceramics $\mathrm{MgAl}_{2} \mathrm{O}_{4}: \mathrm{LiF}$.

observed two bands at $3.1 \mathrm{eV}$ and $3.78 \mathrm{eV}$ which were identified with hole centers at cation vacancies and at anti-site defects, respectively [9]. Considering reaction (1) we can expect the appearance of additional hole centers related to incorporation $\mathrm{Li}^{+}$-ions into the spinel lattice. Due to the specific spinel structure, $\mathrm{Li}^{+}$-ions are distributed on tetrahedral and octahedral cationic sites: $\mathrm{Li}^{+}$-ions in $\mathrm{Mg}$-sites forming $\left(\mathrm{Li}_{\mathrm{Mg}}\right)^{-}$defects or in $\mathrm{Al}$ -sites $\left(\mathrm{Li}_{\mathrm{Al}}\right)^{2-}$ defects (Eq. (1)). The excess of negative charge in the indicated defects leads to a capture of holes and to creation of optically active hole centers, which we have observed after irradiation. The envelope of absorption near $3 \mathrm{eV}$ changes in spectral position from spot to spot, likely because the contribution of $\mathrm{V}$-type centers at isolated cationic vacancies or $\mathrm{Li}^{+}$-containing hole centers have slightly different spectral position of individual absorption bands.

We should also note the large difference in absolute value of absorption in spot (a) compared with that of (b) and (c). Specifically, one may see the small contribution in spectra of spot (a) for the band at $3.8 \mathrm{eV}$, i.e., hole centers at anti-site defects (Fig. 7). Because spot (a) is close to the arc rim we can suppose enhanced diffusion of lithium and fluorine out of the body of monolith during fabrication.

Also, there is some enhancement of the band at $4.75 \mathrm{eV}$ related to $\mathrm{F}^{+}$-centers at irradiation. This can be expected because not all anionic vacancies in the initial samples should capture electrons. Decrease of absorption in the UV range of spectra (the negative difference of optical density) is related to charge change of uncontrolled impurities from initially optically active states to different valence states. The difference absorption spectra between different spots in $\mathrm{X}$-irradiated spinel specimens show that the concentration of $\mathrm{F}^{+}$-centers (anion vacancies) in spot (b) is much higher compared with that of spots (a) and (c); this implies that the cloudy regions contain a higher level of anion vacancies. The concentration of fluorine ions in oxygen sites $\left(\mathrm{F}_{\mathrm{O}}\right)^{+}$decreases in the direction from the arc rim into bulk of ceramic

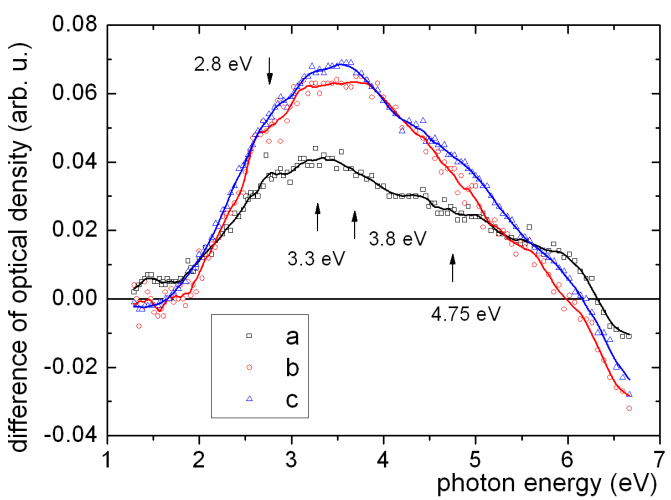

Fig. 7. Radiation induced absorption spectra in different spots (a, b, and c) for transparent ceramics $\mathrm{MgAl}_{2} \mathrm{O}_{4}: \mathrm{LiF}$

disk, supporting the notion that fluorine ions leave more readily at the edges of the sample. Further, in support of this, the larger contribution of $V$-type bands can be seen in irradiated samples for spots (b) and (c) to compare with that of spot (a).

\section{Conclusions}

1. It was revealed that disks of transparent spinel ceramics $\mathrm{MgAl}_{2} \mathrm{O}_{4}: \mathrm{LiF}$ prepared by hot-pressing technique exhibit non-uniformly distributed point defects of different origin. This inhomogeneity is likely a consequence of geometric and environmentally imposed non-uniformities, as well as the variation of the spinel composition during processing.

2. In as-received spinel samples there were observed optical F-type centers related to anionic vacancies, which arise at high temperature during solid chemical reaction of lithium fluorides with spinel.

3. In samples irradiated with X-rays there were observed V-type centers indicating also the existence of cationic vacancies which vary spatially within the spinel part.

4. This research has demonstrated the utility of differential optical spectroscopy to control the degree of perfection during the fabrication of transparent spinel ceramics, and also to predict the behavior of this material in hazard (radiation) fields.

\section{Acknowledgments}

This work was financially supported by the Ministry of Education and Science of Ukraine (Project \#07-13-06). 


\section{References}

[1] D.C. Harris, Materials for Infrared Windows and Domes, Properties and Performance, SPIE Optical Engineering Press, Bellingham, Washington 1999.

[2] M.C.L. Patterson, A.A. DiGiovanni, L. Fehrebacher, D.W. Roy, in: Window and Dome Technologies VIII. SPIE AeroSence, Conf. Proceed., Orlando FL 2003, p. 178.

[3] T.A. Bazilevskaya, V.T. Gritsyna, D.V. Orlinski, L.V. Udalova, A.V. Voitsenya, J. Nucl. Mater. 253 , 133 (1998).

[4] G. Gigle, P. Patel, P. Patterson, D. Blodgett, D. Duncan, D. Hahn, J. Am. Ceram. Soc. 88, 2747 (2005).
[5] H.-J. Kleebe, I.E. Reimanis, R.L. Cook, Ceram. Trans. 157, 61 (2005).

[6] K. Rozenburg, I.E. Reimanis, H.-J. Kleebe, R.L. Cook, J. Am. Ceram. Soc. 90, 2038 (2007).

[7] I.E. Reimanis, H.-J. Kleebe, Int. J. Mat. Res. 98, 1237 (2007).

[8] K. Rozenburg, I.E. Reimanis, H.-J. Kleebe, R.L. Cook, J. Am. Ceram. Soc. 91, 444 (2008).

[9] V.T. Gritsyna, I.V. Afanasyev-Charkin, Yu.G. Kazarinov, K.E. Sickafus, Nucl. Instrun. Methods Phys. Res. B 218, 264 (2004). 